\title{
ON SOME OPTIMIZATION PROBLEM WITH NON-QUADRATIC CRITERION
}

\author{
Adam Kowalewski \\ Institute of Automatics \\ University of Mining and Metallurgy \\ al. Mickiewicza 30, 30-059 Cracow, Poland \\ ako@ia.agh.edu.pl
}

Abstract Various optimization problems for linear parabolic systems with multiple constant time delays are considered. In this paper, we consider an optimal distributed control problem for a linear parabolic system in which multiple constant delays appear in the state equation. Sufficient conditions for the existence of a unique solution of the parabolic time delay equation with the Dirichlet boundary condition are proved. The time horizon $T$ is fixed. Making use of the Lions scheme [9], necessary and sufficient conditions of optimality for the Dirichlet problem with non-quadratic criterion and constrained control are derived.

Keywords: distributed control, parabolic systems, multiple constant delays.

\section{Introduction}

Various optimization problems associated with the optimal control of distributed parabolic systems with time delays appearing in the boundary conditions have been studied recently in Refs. [1]-[8] and [11],[12].

In this paper, we consider an optimal distributed control problem for a linear parabolic system in which multiple constant time delays appear in the state equation.

Such systems constitute in a linear approximation, a universal mathematical model for many diffusion processes.

Sufficient conditions for the existence of a unique solution of such parabolic equations with the Dirichlet conditions are proved. In this paper, we restrict our considerations to the case of the distributed control for the Dirichlet problem. Consequently, we formulate the following op-

\footnotetext{
The original version of this chapter was revised: The copyright line was incorrect. This has been corrected. The Erratum to this chapter is available at DOI: 10.1007/978-0-387-35690-7_44 
timal control problem. We assume that the performance functional has non-quadratic form. Moreover, the time horizon is fixed in our optimization problem. Finally, we impose some constraints on the distributed control . Making use of the Lions framework [9] necessary and sufficient conditions of optimality for the Dirichlet problem with non-quadratic criterion and constrained control are derived.

\section{Existence and uniqueness of solutions}

Consider now the distributed-parameter system described by the following parabolic delay equation

$$
\begin{gathered}
\frac{\partial y}{\partial t}+A(t) y+\sum_{i=1}^{m} b_{i}(x, t) y\left(x, t-h_{i}\right)=v \quad x \in \Omega, t \in(0, T) \\
y\left(x, t^{\prime}\right)=\Phi_{0}\left(x, t^{\prime}\right) \quad x \in \Omega, t^{\prime} \in\left[-h_{m}, 0\right) \\
y(x, 0)=y_{0}(x) \quad x \in \Omega \\
\left.y(x, t ; v)\right|_{\Sigma}=0 \quad x \in \Gamma, t \in(0, T)
\end{gathered}
$$

where: $\Omega \subset R^{n}$ is a bounded, open set with boundary $\Gamma$, which is a $C^{\infty}$-manifold of dimension $n-1$. Locally, $\Omega$ is totally on one side of $\Gamma$,

$$
\begin{array}{cl}
y \equiv y(x, t ; v), & v \equiv v(x, t), \\
Q=\Omega \times(0, T), & \bar{Q}=\bar{\Omega} \times[0, T], \\
Q_{0}=\Omega \times\left[-h_{m}, 0\right), & \Sigma=\Gamma \times(0, T),
\end{array}
$$

$b_{i}$ are given real $C^{\infty}$ functions defined on $\bar{Q}$,

$h_{i}$ are specified positive numbers representing multiple time delays, such

that $0 \leq h_{1}<h_{2}<\ldots<h_{m}$ for $i=1, \ldots, m$,

$\Phi_{0}$ is an initial function defined on $Q_{0}$.

The operator $A(t)$ has the form

$$
A(t) y=-\sum_{i, j=1}^{n} \frac{\partial}{\partial x_{i}}\left(a_{i j}(x, t) \frac{\partial y(x, t)}{\partial x_{j}}\right)
$$

and the functions $a_{i j}(x, t)$ satisfy the condition

$$
\sum_{i, j=1}^{n} a_{i j}(x, t) \Phi_{i} \Phi_{j} \geq \alpha \sum_{i=1}^{n} \Phi_{i}^{2} \quad \alpha>0, \forall(x, t) \in \bar{Q}, \forall \Phi_{i} \in R
$$

where: $a_{i j}(x, t)$ are real $C^{\infty}$ functions defined on $\bar{Q}$ (closure of $Q$ ). The equations (1)-(4) constitute a Dirichlet problem.

First we shall prove sufficient conditions for the existence of a unique solution of the mixed initial-boundary value problem (1)-(4) for the case where $v \in L^{q}(Q), 1<q<\infty$. 
The existence of a unique solution for the mixed initial-boundary value problem (1)-(4) on the cylinder $Q$ can be proved using a constructive method. Using the results of Section 14 ([9], pp. 230-231) we can prove the following result.

Theorem 1.1 Let $y_{0}, \Phi_{0}$ and $v$ be given with $y_{0} \in L^{q}(\Omega), \Phi_{0} \in L^{q}\left(Q_{0}\right)$ and $v \in L^{q}(Q), 1<q<\infty$. Then, there exists a unique solution $y \in L^{q}(Q)$ for the mixed initial-boundary value problem (1)-(4). Moreover, $\frac{\partial y(v)}{\partial x_{i}} \in L^{q}(Q), \quad i=1, \ldots, n$.

Remark 1.1 We refer to [10] for the solution of parabolic problems in $L^{q}, q \neq 2$.

\section{Problem formulation. Optimization theorem}

We shall now formulate the optimal control problem for the Dirichlet problem. Let us denote by $U=L^{q}(Q)$ the space of controls. The time horizon $T$ is fixed in our problem.

The performance functional is given by

$$
I(v)=\lambda_{1} \int_{Q}\left|y(x, t ; v)-z_{d}\right|^{q} d x d t+\lambda_{2}\|v\|_{L^{q}(Q)}^{q}
$$

where: $\lambda_{i} \geq 0, \lambda_{1}+\lambda_{2}>0 ; z_{d}$ is a given element in $L^{q}(Q)$.

Finally, we assume the following constraint on controls $v \in U_{a d}$, where

$$
U_{a d} \text { is a closed, convex subset of } U
$$

Let $y(x, t ; v)$ denote the solution of the mixed initial-boundary value problem (1)-(4) at $(x, t)$ corresponding to a given control $v \in U_{a d}$. We note from the Theorem 1.1 that for any $v \in U_{a d}$ the performance functional (7) is well-defined since $y(v) \in L^{q}(Q)$. The solving of the formulated optimal control problem is equivalent to seeking a $v_{0} \in U_{a d}$ such that $I\left(v_{0}\right) \leq I(v) \quad \forall v \in U_{a d}$.

Remark 2.1 If $q \neq 2, U$ is a reflexive Banach space (but not Hilbert); but as indicated in [9] (p. 8), results of the type of Theorem 1.3 ([9], p.10) remain valid.

Then from the Theorem $1.3([9]$, p. 10) - in the case where $U$ is a reflexive Banach space - it follows that for $\lambda_{2}>0$ a unique optimal control $v_{0}$ exists; moreover, $v_{0}$ is characterized by the following condition

$$
I^{\prime}\left(v_{0}\right) \cdot\left(v-v_{0}\right) \geq 0 \quad \forall v \in U_{\mathrm{ad}}
$$


But from (7), we have for $w \in U$

$$
\left.\begin{array}{l}
I^{\prime}\left(v_{0}\right) w=\left.\frac{d}{d \theta} I\left(v_{0}+\theta w\right)\right|_{\theta=0}= \\
=\lambda_{1} q \int_{Q}\left|y\left(v_{0}\right)-z_{d}\right|^{q-2}\left(y\left(v_{0}\right)-z_{d}\right) \frac{\partial}{\partial v_{0}} y\left(v_{0}\right) w d x d t+ \\
+q \lambda_{2} \int_{Q}\left|v_{0}\right|^{q-2} v_{0} w d x d t
\end{array}\right\}
$$

where

$$
\frac{\partial}{\partial v_{0}} y\left(v_{0}\right) w=\Psi(w)
$$

Then we may verify that

$$
\begin{gathered}
\frac{\partial \Psi(w)}{\partial t}+A \Psi(w)+\sum_{i=1}^{m} b_{i}(x, t) \Psi\left(x, t-h_{i} ; w\right)=w, x \in \Omega, t \in(0, T) \\
\Psi\left(x, t^{\prime}\right)=0 \quad x \in \Omega, t^{\prime} \in\left[-h_{m}, 0\right) \\
\Psi(x, 0 ; w)=0 \quad x \in \Omega \\
\left.\Psi(w)\right|_{\Sigma}=0 \quad x \in \Gamma, t \in(0, T)
\end{gathered}
$$

Consequently, we can express (9) (dividing through by $q$ ) in the following form

$$
\begin{aligned}
& \lambda_{1} \int_{Q}\left|y\left(v_{0}\right)-z_{d}\right|^{q-2}\left(y\left(v_{0}\right)-z_{d}\right) \Psi\left(v-v_{0}\right) d x d t+ \\
& +\lambda_{2} \int_{Q}\left|v_{0}\right|^{q-2} v_{0}\left(v-v_{0}\right) d x d t \geq 0 \quad \forall v \in U_{a d}
\end{aligned}
$$

To simplify (16), we introduce the adjoint equation and for every $v \in U_{a d}$, we define the adjoint variable $p=p(v)=p(x, t ; v)$ as the solution of the equation

$$
\begin{gathered}
-\frac{\partial p(v)}{\partial t}+A^{*}(t) p(v)+\sum_{i=1}^{m} b_{i}\left(x, t+h_{i}\right) p\left(x, t+h_{i} ; v\right)= \\
=\lambda_{1}\left|y(v)-z_{d}\right|^{q-2}\left(y(v)-z_{d}\right), x \in \Omega, t \in\left(0, T-h_{m}\right) \\
-\frac{\partial p(v)}{\partial t}+A^{*}(t) p(v)=\lambda_{1}\left|y(v)-z_{d}\right|^{q-2}\left(y(v)-z_{d}\right), x \in \Omega, t \in\left(T-h_{m}, T\right) \\
p(x, T ; v)=0 \quad x \in \Omega \\
\text { where } p(x, t ; v)=0 \quad x \in \Gamma, t \in(0, T) \\
A^{*}(t) p=-\sum_{i, j=1}^{n} \frac{\partial}{\partial x_{j}}\left(a_{i j}(x, t) \frac{\partial p}{\partial x_{i}}\right)
\end{gathered}
$$

The existence of a unique solution for the problem (17)-(20) on the cylinder $Q$ can be proved using a constructive method. It is easy to notice that for given $z_{d}$ and $v$, problem (17)-(20) can be solved backwards in 
time starting from $t=T$, i.e., first, solving (17)-(20) on the subcylinder $Q_{K}$ and in turn on $Q_{K-1}$, etc. until the procedure covers the whole cylinder $Q$. For this purpose, we may apply Theorem 1.1 (with an obvious change of variables) to problem (17)-(20) (with reversed sense of time, i.e., $t^{\prime}=T-t$ ).

Lemma 2.1 Let the hypothesis of Theorem 1.1 be satisfied. Then, for given $z_{d} \in L^{q}(Q)$ and any $v \in L^{q}(Q)$, there exists a unique solution $p(v) \in L^{q}(Q)$ for the problem (17)-(20). Moreover,

$$
\frac{\partial p(v)}{\partial x_{i}}, \frac{\partial p(v)}{\partial t}, \frac{\partial^{2}}{\partial x_{i} \partial x_{j}} p(v) \in L^{q^{\prime}}(Q)
$$

and

$$
\begin{gathered}
\left|y\left(v_{0}\right)-z_{d}\right|^{q-2}\left(y\left(v_{0}\right)-z_{d}\right) \in L^{q^{\prime}}(Q) \\
\frac{1}{q}+\frac{1}{q^{\prime}}=1 .
\end{gathered}
$$

We simplify (16) using the adjoint equation (17)-(20). For this purpose setting $v=v_{0}$ in (17)-(20), multiplying both sides of (17), (18) by $\Psi\left(v-v_{0}\right)$, then integrating over $\Omega \times\left(0, T-h_{m}\right)$ and $\Omega \times\left(T-h_{m}, T\right)$ respectively and then adding both sides of $(17),(18)$ we get

$$
\begin{gathered}
\lambda_{1} \int_{Q}\left|y\left(v_{0}\right)-z_{d}\right|^{q-2}\left(y\left(v_{0}\right)-z_{d}\right) \Psi\left(v-v_{0}\right) d x d t= \\
=\int_{Q}\left(-\frac{\partial p\left(v_{0}\right)}{\partial t}+A^{*}(t) p\left(v_{0}\right)\right) \Psi\left(v-v_{0}\right) d x d t+ \\
+\sum_{i=1}^{m} \int_{0}^{T-h_{m}} \int_{\Omega} b_{i}\left(x, t+h_{i}\right) p\left(x, t+h_{i} ; v_{0}\right) \Psi\left(v-v_{0}\right) d x d t= \\
=\int_{Q} p\left(v_{0}\right) \frac{\partial}{\partial t} \Psi\left(v-v_{0}\right) d x d t+\int_{Q} A^{*}(t) p\left(v_{0}\right) \Psi\left(v-v_{0}\right) d x d t+ \\
+\sum_{i=1}^{m} \int_{0}^{T-h_{m}} \int_{\Omega} b_{i}\left(x, t+h_{i}\right) p\left(x, t+h_{i} ; v_{0}\right) \Psi\left(v-v_{0}\right) d x d t
\end{gathered}
$$

Using equation (1), the first integral on the right-hand side of (22) can be rewritten as 


$$
\begin{gathered}
\int_{Q} p\left(v_{0}\right) \frac{\partial}{\partial t} \Psi\left(v-v_{0}\right) d x d t=-\int_{Q} p\left(v_{0}\right) A(t) \Psi\left(v-v_{0}\right) d x d t- \\
-\sum_{i=1}^{m} \int_{0}^{T} \int p\left(x, t ; v_{0}\right) b_{i}(x, t) \Psi\left(x, t-h_{i} ; v-v_{0}\right) d x d t+ \\
+\int_{Q} p\left(v_{0}\right)\left(v-v_{0}\right) d x d t=-\int_{Q} p\left(v_{0}\right) A(t) \Psi\left(v-v_{0}\right) d x d t- \\
-\sum_{i=1}^{m} \int_{-h_{i}}^{T-h_{i}} \int_{\Omega} p\left(x, t_{i}+h_{i} ; v_{0}\right) b_{i}\left(x, t_{i}+h_{i}\right) \Psi\left(x, t_{i} ; v-v_{0}\right) d x d t_{i}+ \\
+\int_{Q} p\left(v_{0}\right)\left(v-v_{0}\right) d x d t
\end{gathered}
$$

where: $t_{i}=t-h_{i}$ and $d t=d t_{i}$.

Substituting (23) into (22), after transformations we obtain

$$
\begin{gathered}
\lambda_{1} \int_{Q}\left|y\left(v_{0}\right)-z_{d}\right|^{q-2}\left(y\left(v_{0}\right)-z_{d}\right) \Psi\left(v-v_{0}\right) d x d t= \\
=\int_{Q} p\left(v_{0}\right)\left(v-v_{0}\right) d x d t
\end{gathered}
$$

Substituting (24) into (16) we obtain

$$
\int_{Q}\left(p\left(v_{0}\right)+\lambda_{2}\left|v_{0}\right|^{q-2} v_{0}\right)\left(v-v_{0}\right) d x d t \geq 0, \quad \forall v \in U_{a d}
$$

Theorem 2.1 For the problem (1)-(4) with the performance functional (7) with $z_{d} \in L^{q}(Q)$ and $\lambda_{2}>0$ and with constraints on controls (8), there exists a unique optimal control $v_{0}$ which satisfies the maximum condition (25).

Consider now the particular case where $U_{a d}=L^{q}(Q)$.

Thus the maximum condition (25) is satisfied when

$$
v_{0}=-\frac{1}{\lambda_{2}^{q^{\prime}-1}}|p|^{q^{\prime}-2} p
$$

We must notice that the conditions of optimality derived above (Theorem 2.1) allow us to obtain an analytical formula for the optimal control in particular cases only (e.g. there are no constraints on controls). This results from the following: the determining of the function $p\left(v_{0}\right)$ in the maximum condition (25) is possible from the adjoint equation (17)--(20) if and only if we know $y_{0}$ which corresponds to the control $v_{0}$. These mutual connections make the practical use of the derived optimization formulas difficult. Therefore we resign from the exact determining of the optimal control and we use approximation methods. 
Remark 2.2 We can also consider a non-quadratic form on a Hilbert space: take $U=L^{2}(Q)$; then $y(v) \in L^{q}(Q)$ if $1<q<2$ and hence, in particular, we can consider the performance functional (7) with $1<q<2$.

\section{Conclusions}

The results presented in the paper can be treated as a generalization of the results obtained in [9] onto the case of multiple constant time delays appearing in the state equations.

We can also obtain estimates and a sufficient condition for the boundedness of solutions for such parabolic time delay systems.

Finally, we can consider optimal control problems of hyperbolic systems with multiple time delays appearing in the state equations.

The ideas mentioned above will be developed in forthcoming papers.

\section{Acknowledgments}

The research presented here was carried out within the research programmes University of Mining and Metallurgy, No. 10.10.120.31 and No. 10.10.120.40.

\section{References}

[1] Knowles, G. (1978). Time-optimal control of parabolic systems with boundary conditions involving time delays. Journal of Optimization Theory and Applications, 25:563-574.

[2] Kowalewski, A. (1987). Optimal control with initial state not a priori given and boundary condition involving a delay. Lecture Notes in Control and Information Sciences, Berlin, Heidelberg: Springer-Verlag, 95:94-108,

[3] Kowalewski, A. (1988). Boundary control of distributed parabolic system with boundary condition involving a time-varying lag. International Journal of Control, 48:2233-2248.

[4] Kowalewski, A. (1990). Feedback control for a distributed parabolic system with boundary condition involving a time-varying lag. IMA Journal of Mathematical Control and Information, 7:143-157.

[5] Kowalewski, A. (1990). Minimum time problem for a distributed parabolic system with boundary condition involving a time-varying lag. Archives of Automatics and Remote Control, XXXV, 3-4:145-153.

[6] Kowalewski, A. (1990). Optimality conditions for a parabolic time delay system. Lecture Notes in Control and Information Sciences, Berlin, Heidelberg: SpringerVerlag, 144:174-183.

[7] Kowalewski, A. (1993). Optimal control of parabolic systems with time-varying lags. IMA Journal of Mathematical Control and Information, 10:113-129. 
[8] Kowalewski, A. and Duda, J. (1992). On some optimal control problem for a parabolic system with boundary condition involving a time-varying lag. IMA Journal of Mathematical Control and Information, 9:131-146.

[9] Lions, J.L. (1971). Optimal Control of Systems Governed by Partial Differential Equations. Berlin, Heidelberg: Springer-Verlag.

[10] Solonnikov, V.A. (1965). Mixed problem for a Linear Parabolic Equations in $L^{p}$. Papers of Steklov Mathematical Institute, 83:3-162 (in Russian).

[11] Wang, P.K.C. (1975). Optimal control of parabolic systems wih boundary conditions involving time delays. SIAM Journal of Control, 13:274-293.

[12] Wong, K.H.: (1987). Optimal control computation for parabolic systems with boundary conditions involving time delays. Journal of Optimization Theory and Applications, 53:475-507. 